\title{
Design of a Mobile Bio-diesel Production Facility
}

\author{
B. J. Drake, M. Jacques, D. Binkley, S. Barghi, R. O. Buchal \\ Faculty of Engineering \\ The University of Western Ontario
}

\begin{abstract}
In 2004/2005, a team of mechanical engineering students undertook an interdisciplinary capstone design project to design a mobile bio-diesel production facility capable of converting $500 \mathrm{~L} / \mathrm{h}$ of used vegetable oil or animal tallow into bio-diesel fuel. Bio-diesel fuel has negligible sulfur content and significantly reduces the emission of particulate matter, e.g. soot and carbon monoxide, compared to the combustion of conventional diesel fuel. Furthermore, biodiesel fuel is biodegradable, nontoxic, and can be produced from renewable feedstock. The mobile facility is capable of taking used vegetable oil from different sources and processing the oil while in motion, eliminating costs associated with transportation, land use and construction. A special filter was designed to remove any major particulate matter as well as wax-like substances formed by heating of the cooking oil during its operational life. A rotary vacuum filter was designed to continuously of remove wax and solid particles accumulated on the filter cloth. The wax and solid wastes, which are organic compounds, are readily converted to useful light organic molecules through a subsequent gasification process. A transesterification process was applied using methanol as a solvent and sodium hydroxide as a catalyst. A mix of unrefined bio-diesel fuel and glycerol, which is produced by transestrification, is sent to a glycerol separating tower. The separator was designed to efficiently separate biodiesel fuel from glycerol. The bio-diesel fuel is neutralized by weak acid solution and washed by water to remove impurities. High-speed mixers were designed to create maximum contact between phases for efficient separation. The mobile facility is subject to vibration, which was considered in every aspect of the design. The facility will be powered by bio-diesel fuel, and heat recovery and water recycling were considered to minimize energy requirements. The project culminated in a final design report containing detailed engineering analysis and a comprehensive set of working drawings.
\end{abstract}

